\title{
Current methodologies for molecular screening of thyroid nodules
}

\author{
Elisabetta Macerola, Fulvio Basolo \\ Department of Surgical, Medical, Molecular Pathology and Critical Area, University of Pisa, Pisa, Italy \\ Contributions: (I) Conception and design: F Basolo; (II) Administrative support: All authors; (III) Provision of study materials or patients: All authors; \\ (IV) Collection and assembly of data: E Macerola; (V) Data analysis and interpretation: All authors; (VI) Manuscript writing: All authors; (VII) Final \\ approval of manuscript: All authors. \\ Correspondence to: Prof. Fulvio Basolo, MD, PhD. Department of Surgical, Medical, Molecular Pathology and Critical Area, University of Pisa, via \\ Roma, 57, Pisa 56126, Italy. Email: fulvio.basolo@med.unipi.it.
}

\begin{abstract}
Several molecular tests have been developed for thyroid cytology with the main purpose to define the nature of indeterminate nodules in presurgical phase thus overcoming the limitations of cytological analysis. Molecular tests currently available for thyroid nodules are based on a variety of methodologies and display peculiar strengths and limitations: in this review of the literature they are summarized and critically discussed. The use of molecular diagnostics for thyroid cytology is increasingly widespread and accepted, however large, prospective validation studies are still needed to ultimately demonstrate the clinical efficacy of a wide incorporation of molecular tests into clinical practice.
\end{abstract}

Keywords: Thyroid nodules; thyroid cytology; indeterminate nodules; molecular markers; molecular tests

Submitted Jul 31, 2017. Accepted for publication Aug 06, 2017.

doi: 10.21037 /gs.2017.08.04

View this article at: http://dx.doi.org/10.21037/gs.2017.08.04

\section{Introduction}

Thyroid nodules with indeterminate cytology represent a clinical challenge. It has been reported that approximately $4-43 \%$ of thyroid nodules undergoing fine-needle aspiration (FNA) biopsy are diagnosed as atypia of undetermined significance/follicular lesion of undetermined significance (AUS/FLUS) or follicular neoplasm/suspicious for follicular neoplasm (FN/SFN) according to the Bethesda classification system (1). The risk of malignancy of indeterminate nodules should be always lower than $15 \%$ and $30 \%$ in AUS/FLUS and FN/SFN nodules respectively (2), however, data obtained from real series of cases reported higher malignancy rates $(1,3)$. Surgical treatment for all indeterminate nodules would represent undoubtedly an overly radical treatment in the majority of cases; on the other hand, the risk of malignancy is often too high to safely manage patients with observation only.

Most of indeterminate nodules that prove malignant after surgery are follicular-derived well-differentiated thyroid carcinomas, in particular the follicular-patterned malignancies $(4,5)$ : follicular variant of papillary thyroid carcinoma (FVPTC) and follicular thyroid carcinoma (FTC). FVPTC shows subtle cytonuclear atypia which make them difficult to be definitely recognized on cytology. Instead conventional PTC show marked nuclear atypia and are more rarely diagnosed as indeterminate nodules on cytology; molecularly they are generally $B R A F$-like tumors with $B R A F^{\mathrm{V} 600 \mathrm{E}}$ mutation and RET rearrangement as driver alterations, and display peculiar epi-genetic and genetic patterns (6). By contrast FVPTC are classified as $R A S$-like tumors and have RAS mutations or PPARg translocations (or even $B R A F^{\mathrm{K} 601 \mathrm{E}}$ mutations) as early genetic events (6), even if the infiltrative forms of FVPTC can harbor $B R A F^{\mathrm{V} 600 \mathrm{E}}$ mutations. The general picture of indeterminate cytology is complicated by the fact that the follicular-patterned lesions, namely the malignant FVPTC and FTC, the benign follicular thyroid adenoma and borderline lesions as the noninvasive follicular thyroid neoplasm with papillarylike nuclear features (NIFTP) can share the same molecular alterations (7), such as $R A S$ mutations and PPARg fusions. For these reasons, the role of molecular markers in presurgical settings has been deeply explored.

Much progress has been made in the last 30 years in the 


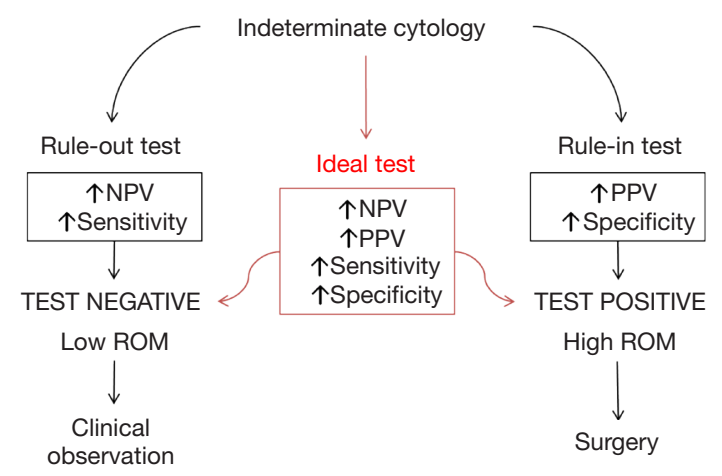

Figure 1 The rule-out and rule-in approaches in the molecular characterization of indeterminate thyroid nodules. The rule-out test has a high negative predictive value (NPV), in other words is informative when a sample results as negative; on the contrary a rule-in test has a high positive predictive value (PPV) and is informative when a sample is positive for the tested markers. The post-test risk of malignancy (indicated as ROM) is therefore reduced in the first case and increased in the second one, however, its value varies according to the pretest prevalence of malignancy in the study population.

understanding of the molecular pathology of thyroid cancer; accordingly, several molecular tests have been developed with the main aim to help cytology evaluation in characterizing indeterminate thyroid nodules. To evaluate the performance of a molecular tests, positive and negative predictive value (PPV and NPV) can be extrapolated. Predictive values are calculated on the basis of specificity and sensitivity of a test, and should always take into account the specific pretest probability of malignancy observed in each study cohort. For this reason, PPV and NPV of a molecular test can vary when it is performed in different institutions. A test with high PPV can be generally defined as a rule-in test; on the contrary, a high NPV indicates that the test should be powerful in ruling-out malignancy (Figure 1).

To be effectively applied on thyroid cytology, a good rule-out test should decrease the risk of malignancy of an indeterminate nodule so that it is comparable with the risk observed in benign cytology—and therefore the test can likely reduce the rate of unnecessary surgeries. For example, a test with sensitivity of $90 \%$ and specificity of $25 \%$ could roughly be a good candidate as a rule-out test. In a population with pre-test risk of malignancy of $15 \%$, NPV would be of $93 \%$ and the post-test risk of malignancy would be reduced to $7 \%$, which is comparable with the risk of benign cytology. In a population with a pre-test prevalence of malignancy of $30 \%$, the same test would have a NPV of $85 \%$ and therefore the risk of malignancy would remain relatively high $(15 \%)$, still comparable with that of indeterminate cytology. Therefore, in the latter case, the test would not work as well as expected and would probably prove not worthwhile.

The molecular analysis of thyroid cytology specimens can be generally performed on DNA/RNA extracted from stained smears or from aspirates collected in nucleic acids preservative solutions. Molecular tests can be based on a variety of methodologies, starting from standard immunocytochemistry (ICC) analysis to more advanced and multi-target techniques. The different techniques available for thyroid cytology are summarized in the next paragraphs along with the results obtained with the tested molecular markers.

\section{ICC}

The expression of specific proteins can be evaluated by ICC on cytological smears with the aim to support diagnosis. Several markers have been proposed during the last 20 years (8-12). Galectin-3 and HBME-1 are the markers with the highest specificity, but they showed low sensitivity, thus a negative immunostaining does not exclude malignancy. Other proposed markers are cytokeratin 19, fibronectin-1 and CD44. In order to increase the accuracy of fine-needle aspiration cytology (FNAC) evaluation, several groups have been investigating the usefulness of panels with multiple ICC markers and obtained encouraging results $(8,12)$. The ICC has several advantages, it represents a cost-effective approach and requires standard lab equipment, therefore it could be performed as a routine test. However, ICC analysis is often limited by the scarcity of fine-needle aspiration (FNA) material (in particular for the evaluation of ICC panels), by the lack of reproducibility among laboratories and the interobserver variability in the interpretation of results.

\section{Molecular tests based on mRNA and microRNA (miRNA) expression analysis}

The altered expression of specific mRNAs such as fibronectin-1, TIMP1 and E-selectin $(13,14)$ has been reported in thyroid tumors; however, to date no single mRNA has achieved satisfactorily high sensitivity and specificity to be effectively applied as potential marker on thyroid 
cytology. For this reason, several studies have recently investigated the potential role of mRNA panels rather than the analysis of expression of single genes. The hypothesis is that the analysis of mRNA expression patterns leads to the identification of peculiar phenotypic signatures that can be associated to specific thyroid lesions.

The main commercial molecular test based on this strategy is the Afirma gene expression classifier (GEC) by Veracyte Inc. (South San Francisco, CA, USA). Afirma test is based on a microarray analysis of mRNA of 167 genes by a two-step process: the first step includes the evaluation of a 25-gene panel developed to recognize some less frequent neoplastic pathologies of the thyroid, such as medullary thyroid carcinoma or metastatic lesions; the second step involves the analysis of the main 142-gene panel. The 25gene panel represents a preliminary screening to select the nodules that can be successfully classified by using the combined expression of the 142 genes. The Afirma is based on a proprietary classification algorithm that has been trained by testing cytological samples with known histological outcome. The entire system is organized to recognize benign nodules (rule-out approach), and labels each tested sample with a "benign" or "suspicious" result. mRNA is purified from FNA material obtained through two dedicated FNA passes and collected in a preservative solution; the company requires additional FNA material for performing a centralized cytological evaluation and only the nodules diagnosed as Bethesda III and IV are definitely accepted for Afirma test.

The validation study, a prospective clinical trial conducted blindly, included a total of 265 nodules with indeterminate cytology and surgical follow-up (15). The study revealed that a "benign" GEC result was able to reduce the risk of malignancy of a nodule thanks to the high NPV of the test (94\%). This could lead to a reduction of rate of unnecessary surgeries, thus making safer a conservative approach. On the other hand, a "suspicious" result is not informative, since the test demonstrated low specificity and PPV (52\% and 37\%, respectively). As a matter of facts several subsequent studies evaluating GEC test performance on indeterminate nodules confirmed the results of the validation study in terms of high sensitivity (83-100\%) and low specificity (7-60\%) and legitimated the efficacy of Afirma GEC as a powerful ruleout test $(16,17)$. The sensitivity of a test is calculated as the ratio between the true positive cases (GEC "suspicious" and malignant on histology) and the true positive plus the false negative cases ("benign" on GEC and malignant on final histology). The majority of post-validation studies included a low number of false negative, or even a total absence of such cases, but often this is due to the extremely low rate of GEC "benign" nodules undergoing diagnostic surgery and could likely lead to underestimate the prevalence of malignancy among them.

In any case, as for each other molecular test, the performance of GEC has to be calculated in the context of the institutional pretest risk of malignancy among indeterminate cytology (18). For example, Roychoudhury and colleagues reported that in their institution cytology alone was more powerful than Afirma GEC in predicting malignancy in the context of nodules cytologically diagnosed as suspicious for follicular neoplasm (Bethesda IV) and therefore they raised the question about the utility of GEC in this specific setting (19).

Regarding the new pathological entity of NIFTP, "noninvasive follicular neoplasm with papillary-like nuclear features", few studies have so far reported the performance of GEC on nodules histologically diagnosed as NIFTP and it seems that in most of cases they are classified as GEC "suspicious" (20-23). Since the majority of NIFTPs arise as cytologically indeterminate nodules $(24,25)$, this may constitute an issue that raises the need for further studies evaluating GEC results also in the context of other risk factors (genetic alterations, ultrasound features, nuclear atypia and so on).

A new promising technology already tested on thyroid cytology for the evaluation of mRNA expression is the nCounter NanoString system (26). This methodology is based on the hybridization of target mRNA and/or miRNA molecules with specific barcoded probes and performs a direct digital count of the hybridized complexes. This allows to skip both retrotranscription and amplification steps. Moreover, the reaction can be multiplexed (up to 800 targets in a single well) maintaining a high level of reproducibility $(27,28)$. The nCounter system could be useful in the preoperative assessment of thyroid cytology (26), but no ready-to-use panels specifically designed for thyroid cytology have been developed so far.

miRNA are small non-coding RNA molecules that regulate the expression at post-transcriptional level mainly by annealing with a specific mRNA target, leading to its degradation or to the inhibition of its translation (29). Specific miRNA signatures have been described in (human) cancer and correlated with pathological features of tumors, with prognosis and response to therapies (30). Several studies investigated the role of miRNA in thyroid cancer (27,31-33), and at least two miRNA-based molecular tests 
have been specifically evaluated on indeterminate cytology: the RosettaGX Reveal test (Rosetta Genomics company, Philadelphia, PA, USA) and the ThyraMIR test (Interpace Diagnostics Group Inc., Parsippany, NJ, USA). RosettaGX Reveal works on RNA obtained from one of the stained smears routinely employed for cytology diagnosis. The test is based on a quantitative retrotranscription PCR that measures the expression level of 24 miRNAs. Then classification algorithms use expression data and give three possible results, "benign", "suspicious for malignancy" or "positive for medullary marker" (34). RosettaGX test has been clinically validated in two studies $(34,35)$ showing high sensitivity, specificity and NPV. The ThyraMIR test evaluates the expression level of 10 miRNAs by quantitative retro-transcription PCR. The test algorithm, conveniently trained on thyroid specimens with known surgical outcome, reports samples as "positive" or "negative". This test is intended to perform on nodules negative for the 7-gene mutation panel, which will be discussed in the next paragraphs, with the aim to combine the advantages of both type of tests and improve the overall diagnostic sensitivity in indeterminate cytology (36).

\section{Methods for detecting of genotype alterations}

The study of the molecular landscape of thyroid cancer allowed the identification of a series of somatic alterations, such as gene mutations and rearrangements, directly involved in thyroid tumorigenesis. The most frequent genotype alterations detected in well-differentiated thyroid cancer-therefore the most useful in the settings of the indeterminate cytology-include point mutations in $B R A F$, $N R A S, H R A S$ and KRAS genes, and the RET/PTC and $P A X 8 / P P A R g$ translocations (37). More rarely mutations in TP53, PIK3CA and in the promoter of TERT gene can be detected on indeterminate FNA specimens, also in coexistence with other driver mutations, and indicate highrisk lesion (38).

Generally, the detection of a single mutation or translocation in indeterminate cytology is indicative for malignancy, however these alterations are not sensitive enough to exclude malignancy when mutational test is negative. Therefore, they work well as a rule-in approach. During the last twenty years, hundreds of studies have been investigating the clinical role of specific molecular alterations, such as $B R A F$ mutations, detected on indeterminate thyroid cytology (39). An increasingly common strategy is to consider panels of markers simultaneously rather than single-gene mutations.
The role of a restricted panel analyzing the most frequent oncogenic mutations and translocations (the so called 7-gene panel: BRAF, NRAS, HRAS, KRAS, RET/PTC1, RET/ PTC3, PAX8/PPARg) has been studied in indeterminate cytology by several authors $(37,40-42)$ and obtained good performance in terms of specificity and PPV. It requires the extraction of both DNA and RNA from one dedicated FNA pass collected in a preservative solution. Initially this test was based on melting curve analysis, Sanger sequencing and real-time PCR (37) but now Interpace Diagnostics offers a NGS-based analysis of a panel of 8 genes (the oncogene PIK3CA was added) and commercialized as the ThyGenX test. Labourier and colleagues investigated the diagnostic value of the ThyGenX/ThyraMIR combined strategy and demonstrated that this combination increases the NPV, thus improving the overall test performance on indeterminate nodules (36).

The ThyroSeq v. 2 is a NGS-based test analyzing a comprehensive panel of genes specific for thyroid cancer. It is commercialized by CBLPath Inc. (Rye Brook, NY, USA), while molecular analysis is centralized in the Division of Molecular and Genomic Pathology at the University of Pittsburgh Medical Center (Pittsburgh, PA, USA). ThyroSeq v.2 includes (I) the analysis at DNA level of 14 genes screened for point mutations (BRAF, RAS genes, PIK3CA, TP53, RET, promoter of TERT and others); and (II) the analysis of mRNA to evaluate 42 gene fusions and the expression levels of 16 genes. In the validation study, which included a total of 143 indeterminate nodules with surgical outcome, this extended panel showed promising results, since 35 out of 42 mutation positive cases $(83 \%)$ and only 4 out of 101 mutation-negative nodules (4\%) proved malignant on final histology, with PPV of $83 \%$ and NPV of $96 \%$ (43). Therefore, this preliminary study showed that ThyroSeq could work as both rule-in and rule-out test. The gene panel was extended beyond the canonical 7 genes with the main purpose of increasing its sensitivity, however some of the added markers contributed to reduce the test specificity, being detected also in benign thyroid neoplasms (TSHR, EIF1AX, THADA fusions) (44). As a matter of fact, in a recent study conducted by Valderrabano and colleagues on Bethesda III and IV nodules, ThyroSeq showed a lower PPV compared to the previous studies ( $42 \%$ and $33 \%$ respectively considering NIFTPs as malignant or benign) (45). Undoubtedly ThyroSeq analysis can help in distinguishing low- from high-risk thyroid follicular neoplasms, being able to detect secondary molecular alterations associated with tumor aggressiveness (such 
as TERT promoter mutations). However, since only few studies in literature explored the usefulness of ThyroSeq in indeterminate cytology, further independent research is warranted to better explore its clinical role.

The ThyroSeq panel has been specifically designed for thyroid cytology, however other NGS panels have been tested on thyroid nodules specimens. The Ion AmpliSeq Cancer Hotspot Panel v2 (Thermo Fisher Scientific Inc., Waltham, MA, USA) that analyzes oncogenic mutations in 50 genes was tested by Le Mercier and collaborators in 34 indeterminate nodules with known surgical outcome (46). Even if the authors obtained encouraging results $(63 \%$ and $92 \%$ PPV and NPV, respectively), the study was conducted on a limited cohort of patients. Similarly, Fuller et al. investigated mutations and gene fusion in $24 \mathrm{FNA}$ specimens, among which only nine were indeterminate on cytology (47). In these nine samples authors found no mutations. Therefore, the usefulness of this kind of panels on thyroid cytology should be investigated in more details.

\section{Final considerations}

Each molecular test performed on indeterminate thyroid cytology has either strengths and limitations. Before incorporating any type of molecular testing into a specific clinical context, several factors should be taken into account: (I) the pretest prevalence of malignancy observed in each institution, which can change depending on slight differences in the pathological criteria for the diagnosis of indeterminate nodules; (II) an institutional cost/benefit evaluation, so is the cost afforded for molecular testing really balanced by a real advantage in terms of sanitary expenses? And what kind of molecular test can be more convenient? (III) The possibility of an active interaction between clinicians and pathologists in defining subgroups of nodules to manage efficiently with the adjunct of a molecular approach.

All these factors taken together determine the real usefulness of molecular characterization of nodules in ultimately reducing the overtreatment of benign cytology.

\section{Acknowledgements}

None.

\section{Footnote}

Conflicts of Interest: The authors have no conflicts of interest to declare.

\section{References}

1. Haugen BR, Alexander EK, Bible KC, et al. 2015 American Thyroid Association Management Guidelines for Adult Patients with Thyroid Nodules and Differentiated Thyroid Cancer: The American Thyroid Association Guidelines Task Force on Thyroid Nodules and Differentiated Thyroid Cancer. Thyroid 2016;26:1-133.

2. Cibas ES, Ali SZ. The Bethesda System for Reporting Thyroid Cytopathology. Thyroid 2009;19:1159-65.

3. Shrestha RT, Evasovich MR, Amin K, et al. Correlation Between Histological Diagnosis and Mutational Panel Testing of Thyroid Nodules: A Two-Year Institutional Experience. Thyroid 2016;26:1068-76.

4. Brophy C, Mehanna R, McCarthy J, et al. Outcome of Subclassification of Indeterminate (Thy-3) Thyroid Cytology into Thy-3a and Thy-3f. Eur Thyroid J 2015;4:246-51.

5. Proietti A, Sartori C, Borrelli N, et al. Follicular-derived neoplasms: morphometric and genetic differences. J Endocrinol Invest 2013;36:1055-61.

6. Cancer Genome Atlas Research Network. Integrated genomic characterization of papillary thyroid carcinoma. Cell 2014;159:676-90.

7. Nikiforov YE, Seethala RR, Tallini G, et al. Nomenclature Revision for Encapsulated Follicular Variant of Papillary Thyroid Carcinoma: A Paradigm Shift to Reduce Overtreatment of Indolent Tumors. JAMA Oncol 2016;2:1023-9.

8. Bizzarro T, Martini M, Marrocco C, et al. The Role of CD56 in Thyroid Fine Needle Aspiration Cytology: A Pilot Study Performed on Liquid Based Cytology. PloS One 2015;10:e0132939.

9. Cochand-Priollet B, Dahan H, Laloi-Michelin M, et al. Immunocytochemistry with cytokeratin 19 and anti-human mesothelial cell antibody (HBME1) increases the diagnostic accuracy of thyroid fine-needle aspirations: preliminary report of 150 liquid-based fineneedle aspirations with histological control. Thyroid 2011;21:1067-73.

10. Hashimoto K, Morimoto A, Kato M, et al. Immunocytochemical analysis for differential diagnosis of thyroid lesions using liquid-based cytology. Nagoya J Med Sci 2011;73:15-24.

11. Rossi ED, Raffaelli M, Minimo C, et al. Immunocytochemical evaluation of thyroid neoplasms on 
thin-layer smears from fine-needle aspiration biopsies. Cancer 2005;105:87-95.

12. Saggiorato E, De Pompa R, Volante M, et al. Characterization of thyroid 'follicular neoplasms' in fineneedle aspiration cytological specimens using a panel of immunohistochemical markers: a proposal for clinical application. Endocr Relat Cancer 2005;12:305-17.

13. Griffith OL, Melck A, Jones SJ, et al. Meta-analysis and meta-review of thyroid cancer gene expression profiling studies identifies important diagnostic biomarkers. J Clin Oncol 2006;24:5043-51.

14. Miccoli P, Torregrossa L, Borrelli N, et al. E-selectin expression and BRAF status in papillary thyroid carcinomas: Correlation with clinicopathologic features. Surgery 2014;156:1550-7; discussion 1557-8.

15. Alexander EK, Kennedy GC, Baloch ZW, et al. Preoperative diagnosis of benign thyroid nodules with indeterminate cytology. N Engl J Med 2012;367:705-15.

16. Kloos RT. Molecular Profiling of Thyroid Nodules: Current Role for the Afirma Gene Expression Classifier on Clinical Decision Making. Mol Imaging Radionucl Ther 2017;26:36-49.

17. Nishino M. Molecular cytopathology for thyroid nodules: A review of methodology and test performance. Cancer Cytopathol 2016;124:14-27.

18. Valderrabano P, Leon ME, Centeno BA, et al. Institutional prevalence of malignancy of indeterminate thyroid cytology is necessary but insufficient to accurately interpret molecular marker tests. Eur J Endocrinol 2016;174:621-9.

19. Roychoudhury S, Klein M, Souza F, et al. How "suspicious" is that nodule? Review of "suspicious" Afirma gene expression classifier in high risk thyroid nodules. Diagn Cytopathol 2017;45:308-11.

20. Baca SC, Wong KS, Strickland KC, et al. Qualifiers of atypia in the cytologic diagnosis of thyroid nodules are associated with different Afirma gene expression classifier results and clinical outcomes. Cancer cytopathol 2017;125:313-22.

21. Hang JF, Westra WH, Cooper DS, et al. The impact of noninvasive follicular thyroid neoplasm with papillary-like nuclear features on the performance of the Afirma gene expression classifier. Cancer Cytopathol 2017;125:683-91.

22. Jiang XS, Harrison GP, Datto MB. Young Investigator Challenge: Molecular testing in noninvasive follicular thyroid neoplasm with papillary-like nuclear features. Cancer Cytopathol 2016;124:893-900.

23. Samulski TD, LiVolsi VA, Wong LQ, et al. Usage trends and performance characteristics of a "gene expression classifier" in the management of thyroid nodules: An institutional experience. Diagn Cytopathol 2016;44:867-73.

24. Ibrahim AA, Wu HH. Fine-Needle Aspiration Cytology of Noninvasive Follicular Variant of Papillary Thyroid Carcinoma Is Cytomorphologically Distinct From the Invasive Counterpart. Am J Clin Pathol 2016;146:373-7.

25. Maletta F, Massa F, Torregrossa L, et al. Cytological features of "noninvasive follicular thyroid neoplasm with papillary-like nuclear features" and their correlation with tumor histology. Hum Pathol 2016;54:134-42.

26. Giannini R, Torregrossa L, Gottardi S, et al. Digital gene expression profiling of a series of cytologically indeterminate thyroid nodules. Cancer Cytopathol 2015;123:461-70.

27. Borrelli N, Denaro M, Ugolini C, et al. miRNA expression profiling of 'noninvasive follicular thyroid neoplasms with papillary-like nuclear features' compared with adenomas and infiltrative follicular variants of papillary thyroid carcinomas. Mod Pathol 2017;30:39-51.

28. Veldman-Jones MH, Brant R, Rooney C, et al. Evaluating Robustness and Sensitivity of the NanoString Technologies nCounter Platform to Enable Multiplexed Gene Expression Analysis of Clinical Samples. Cancer Res 2015;75:2587-93.

29. Calin GA, Croce CM. MicroRNA signatures in human cancers. Nat Rev Cancer 2006;6:857-66.

30. Li MH, Fu SB, Xiao HS. Genome-wide analysis of microRNA and mRNA expression signatures in cancer. Acta Pharmacol Sin 2015;36:1200-11.

31. Keutgen XM, Filicori F, Crowley MJ, et al. A panel of four miRNAs accurately differentiates malignant from benign indeterminate thyroid lesions on fine needle aspiration. Clin Cancer Res 2012;18:2032-8.

32. Pallante P, Battista S, Pierantoni GM, et al. Deregulation of microRNA expression in thyroid neoplasias. Nat Rev Endocrinol 2014;10:88-101.

33. Zhang Y, Zhong Q, Chen X, et al. Diagnostic value of microRNAs in discriminating malignant thyroid nodules from benign ones on fine-needle aspiration samples. Tumour Biol 2014;35:9343-53.

34. Benjamin H, Schnitzer-Perlman T, Shtabsky A, et al. Analytical validity of a microRNA-based assay for diagnosing indeterminate thyroid FNA smears from routinely prepared cytology slides. Cancer Cytopathol 2016;124:711-21.

35. Lithwick-Yanai G, Dromi N, Shtabsky A, et al. Multicentre validation of a microRNA-based assay for diagnosing indeterminate thyroid nodules utilising fine needle aspirate 
smears. J Clin Pathol 2017;70:500-7.

36. Labourier E, Shifrin A, Busseniers AE, et al. Molecular Testing for miRNA, mRNA, and DNA on Fine-Needle Aspiration Improves the Preoperative Diagnosis of Thyroid Nodules With Indeterminate Cytology. J Clin Endocrinol Metab 2015;100:2743-50.

37. Nikiforov YE, Steward DL, Robinson-Smith TM, et al. Molecular testing for mutations in improving the fineneedle aspiration diagnosis of thyroid nodules. J Clin Endocrinol Metab 2009;94:2092-8.

38. Hsiao SJ, Nikiforov YE. Molecular approaches to thyroid cancer diagnosis. Endocr Relat Cancer 2014;21:T301-13.

39. Su X, Jiang X, Xu X, et al. Diagnostic value of BRAF (V600E)-mutation analysis in fine-needle aspiration of thyroid nodules: a meta-analysis. Onco Targets Ther 2016;9:2495-509.

40. Beaudenon-Huibregtse S, Alexander EK, Guttler RB, et al. Centralized molecular testing for oncogenic gene mutations complements the local cytopathologic diagnosis of thyroid nodules. Thyroid 2014;24:1479-87.

41. Cantara S, Capezzone M, Marchisotta S, et al. Impact of proto-oncogene mutation detection in cytological specimens from thyroid nodules improves the diagnostic accuracy of cytology. J Clin Endocrinol Metab 2010;95:1365-9.

42. Nikiforov YE, Ohori NP, Hodak SP, et al. Impact of mutational testing on the diagnosis and management of

Cite this article as: Macerola E, Basolo F. Current methodologies for molecular screening of thyroid nodules. Gland Surg 2018;7(Suppl 1):S1-S7. doi: 10.21037/gs.2017.08.04 patients with cytologically indeterminate thyroid nodules: a prospective analysis of 1056 FNA samples. J Clin Endocrinol Metab 2011;96:3390-7.

43. Nikiforov YE, Carty SE, Chiosea SI, et al. Highly accurate diagnosis of cancer in thyroid nodules with follicular neoplasm/suspicious for a follicular neoplasm cytology by ThyroSeq v2 next-generation sequencing assay. Cancer 2014;120:3627-34.

44. Nikiforov YE, Carty SE, Chiosea SI, et al. Impact of the Multi-Gene ThyroSeq Next-Generation Sequencing Assay on Cancer Diagnosis in Thyroid Nodules with Atypia of Undetermined Significance/Follicular Lesion of Undetermined Significance Cytology. Thyroid 2015;25:1217-23.

45. Valderrabano P, Khazai L, Leon ME, et al. Evaluation of ThyroSeq v2 performance in thyroid nodules with indeterminate cytology. Endocr Relat Cancer 2017;24:127-36.

46. Le Mercier M, D'Haene N, De Neve N, et al. Nextgeneration sequencing improves the diagnosis of thyroid FNA specimens with indeterminate cytology. Histopathology 2015;66:215-24.

47. Fuller MY, Mody D, Hull A, et al. Next-Generation Sequencing Identifies Gene Mutations That Are Predictive of Malignancy in Residual Needle Rinses Collected From Fine-Needle Aspirations of Thyroid Nodules. Arch Pathol Lab Med 2018;142:178-83. 